\title{
LABYRINTHUS HIC HABITAT MINOTAURUS
}

\author{
Beethovens Grande Fugue und unser Angewiesensein auf \\ Orientierung
}

Stefan Berg

«Das Monstrum aller Quartettmusik» Thomas Mann, Doktor Faustus

In den Fugengängen von Beethovens Grande Fugue op. 133 haust etwas Monströses wie der Minotaurus im Labyrinth. Interpreten und Hörer berichten vom Herumirren in den Winkeln dieses tönenden Geflechts, von Begegnungen mit etwas, das irgendwo dort im Inneren auf der Lauer liegt. Nur einem Hörer, der dem Helden Theseus gliche, wäre es vergönnt, mit sicheren Schritten auch in die tiefste Kammer des Klanges vorzudringen, den irren Herrn des Kontrapunkts zu besiegen und dank eines musikalischen Ariadnefadens ans Tageslicht zurückzufinden. Unsereinem bleibt nur waghalsige Annäherung - und die Hoffnung, in Eile noch Weniges skizzieren zu können, bevor auch wir dort drinnen zur Beute werden.

Damit wir nicht ganz unvorbereitet in die Grande Fugue hineintappen, machen wir uns zunächst klar, was uns erwartet.

\section{Das Labyrinth und die Eskalation des Orientierungsbedürfnisses}

Wer die Pforte des Labyrinths überschreitet, dessen Orientierungsfähigkeit wird auf die Probe gestellt. Werner Stegmaier schreibt, Orientierung sei die «Leistung, sich in wechselnden Situationen zurechtzufinden» ${ }^{1}$. Folglich ist das Labyrinth eine krisenhafte Situation, in der die gewohnten Strategien des Zurechtfindens versagen und das Bedürfnis nach Orientierung eskaliert. Dies ergibt sich nicht zufällig: Zwar kann uns jede verwirrende Situation zum Labyrinth im übertragenen Sinn werden, doch ein echtes Labyrinth wurde entworfen, um eine solche Eskalation herbeizuführen.

\footnotetext{
W. Stegmaier, Einleitung, in: Orientierung. Philosophische Perspektiven, hg.v. W. Stegmaier, Frankfurt a.M. 2005, 14-50, hier: 16.
} 
Um dies genauer zu verstehen, bedarf es einerVorstellung von gelingender Orientierung. Diese kann bestimmt werden als ein fünfstelliger personaler Vollzug: Jemand (das Orientierungssubjekt) ortet sich (der Ortungsvollzug) in etwas (dem Orientierungsraum) anhand von etwas (des Bezugspunkts) in Rekurs auf etwas (die Ordnung). So kann sich z.B. der Wanderer im Gebirge orten, indem er angesichts eines markanten Geländepunkts in seiner Nähe (z.B. einer Brücke) auf die Ordnung einer Wanderkarte rekurriert. Diese enthält eine Auswahl und Anordnung von Bezugspunkten, die für das Wandern relevant sind (Brücken und Gewässer, aber nicht Kühe und Autos). Findet der Wanderer den anvisierten Bezugspunkt auf der Karte, so kann er seine eigene Position im Orientierungsraum bestimmen. Dadurch erschliesst sich ihm die Umgebung, und es eröffnen sich neue Handlungsmöglichkeiten. Doch Ordnungen begegnen nicht nur in Gestalt von Landkarten. Als solche kommt alles in Betracht, was Phänomene derWelt (also mögliche Bezugspunkte: Gegenstände, Personen, Handlungen usw.) in bestimmter Auswahl und Anordnung enthält - sei es als Erfahrungsordnung (Anordnung von Phänomenen, die in der Erinnerung bewahrt bleibt), als Konventionsordnung (übliche Anordnung) oder als Gesetzesordnung (kodifiziert vorgeschriebene Anordnung). In unserem alltäglichen und ausseralltäglichen Zurechtfinden in der Welt rekurrieren wir auf verschiedenste dieser Ordnungen, wobei sich eingespielte Orientierungsstrategien herausbilden. Und weil wir uns auch in unseren Handlungen, mit denen wir gestaltend in die Welt eingreifen, orientieren müssen, hinterlassen wir in ihr Spuren dieser Strategien.

Das Labyrinth stört diese eingespielten Ordnungsrekurse. Dabei lassen sich drei Methoden labyrinthischer Desorientierung benennen:

1. der Entzug von Bezugspunkten

a) durch Maximierung redundanter Strukturen

b) durch Minimierung markanter Strukturen

2. die irreführende Setzung von Bezugspunkten

a) Bezugspunkte, von denen auf mehrere Ordnungen rekurriert werden kann

b) Bezugspunkte, von denen nur auf eine inadäquate Ordnung rekurriert werden kann

3. die Isolierung von Bezugspunkten

Die erste Methode bedingt, dass im Labyrinth Gänge und Kreuzungen miteinander verwechselt werden können. Aufgrund dieses Fehlens von Markanz, muss an einer Kreuzung zwischen vermeintlich iden- 
tischen Optionen gewählt werden. Die zweite Methode lässt zwar Bezugspunkte $\mathrm{zu}$, doch lockt sie mit ihnen bewusst auf eine falsche Fährte, etwa indem sie Ausgänge vortäuscht oder scheinbar markante Strukturen stillschweigend vervielfältigt. Die dritte Methode isoliert Bezugspunkte. Uns fällt Orientierung dann leichter, wenn wir mehrere Bezugspunkte zugleich anpeilen können. Wenn etwa neben der Brücke noch ein Gebäude steht, ist dies in der Ordnung der Landkarte leichter zu identifizieren. Das Labyrinth unterbindet dergleichen, indem es seinem Besucher Perspektiven vorenthält, die eine gewisse Übersicht eröffnen könnten - etwa durch die Nivellierung des Terrains.

Zur Illustration zwei französische Gartenlabyrinthe:

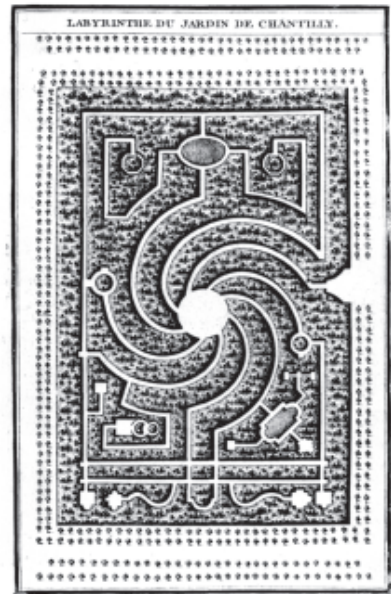

Das Labyrinth im Jardin de Chantilly

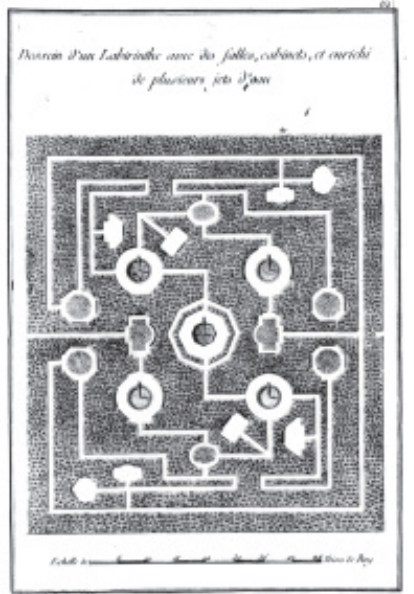

Entwurf eines Labyrinth von P.J. Galimard

Noch ein Gedanke zur gängigen Differenzierung zwischen «echten〉 Labyrinthen und Irrgärten: Erstere seien kreuzungsfrei, und nur letztere enthielten Wahlmöglichkeiten. Diese Unterscheidung ist nicht zuletzt deshalb problematisch, weil Theseus am locus classicus labyrinthicus kaum einen Ariadnefaden benötigt hätte, wenn es sich um einen kreuzungsfreien Weg gehandelt hätte. Ich würde daher vorschlagen, zwischen räumlichen und graphischen Labyrinthen $\mathrm{zu}$ unterscheiden. Während im räumlichen Labyrinth die genannten 
Methoden im dreidimensionalen Raum zur Anwendung kommen, werden sie im graphischen auf die visuelle Ebene übertragen. Das desorientierende Prinzip bleibt identisch, gleichgültig, ob man sich mit dem ganzen Leib im Gewirr von Gängen verirrt oder nur mit den Augen im Gewirr von Linien.

\section{Musikalische Labyrinthe}

Es wurde immer wieder versucht, die Methoden labyrinthischer Desorientierung im Medium der Musik anzuwenden. ${ }^{2}$ Musikalische Labyrinthe sind demnach Klangräume, in denen sich eine Eskalation von musikalischen Orientierungsbedürfnissen ereignet. Dabei wird der Rekurs von musikalischen Bezugspunkten (Akkorde, Melodien, Rhythmen etc.) auf musikalische Ordnungen gestört, also auf die dem Hörer - bewusst oder unbewusst - vertrauten Ordnungen der Melodik, Harmonik, Kontrapunktion, Formenlehre usw. Die Methoden labyrinthischer Desorientierung ereignen sich hier im Medium des Klanges und der Zeit.

Anders als ein Labyrinth in Gestalt von Kieswegen und Hecken entsteht ein musikalisches Labyrinth erst im Moment seiner Klangwerdung - genauer gesagt sogar erst, wenn ein kompetenter Hörer gegenwärtig ist. Denn weil sich das klingende Labyrinth erst durch die Störungen der Rekurse konstituiert, muss eine gewisse musikalische Orientierungskompetenz gegeben sein, die irritiert werden kann. Das heisst aber auch, dass jedes intentional entworfene musikalische Labyrinth auf bestimmte Orientierungsstrategien hin angelegt ist - in der Regel diejenigen, die im unmittelbaren historischen und regionalen Kontext gängig sind.

Mit der Medialität des musikalischen Labyrinths geht aber noch ein Weiteres einher: Das musikalische Labyrinth kennt keine wirklichen Kreuzungen. Der Hörer kann - von jüngeren Versuchen, Interaktion zu erzeugen, einmal abgesehen - nicht in das musikalische Geschehen eingreifen und hat daher auch keine echten Entscheidungsoptionen. Zwar gewährt ihm etwa ein mehrdeutiger Akkord die Möglichkeit, auf diese oder jene harmonische Ordnung zu rekurrieren, doch nur einen Moment später wird eine der Optionen faktisch erklingen, womit sich das vermeintlich Mehrdeutige ex post als schon lange ge-

2 Insbesondere in Barock und Moderne; verwiesen sei stellvertretend auf die Kompositionen von M. Marais (Le Labyrinthe), J.C.F. Fischer (Ariadne musica), P. Locatelli (Laberinto armonico, aus: L'arte del violino op. 3), Hans-Jürgen von Bose (Labyrinth I und II) und Hans-Werner Henze (Labyrinth). 
stellte Weiche erweist. Die Temporalität der Musik bedingt demnach, dass multioptionale Kreuzungen für den Hörer in der Regel nur ex ante bestehen können. Musikalische Labyrinthe sind daher auf diese Perspektive ausgerichtet. Dies lässt sich auch als temporale Isolation von Bezugspunkten im Sinn der dritten Methode labyrinthischer Desorientierung verstehen.

Um die Idee musikalischer Labyrinthe weiter zu präzisieren, möchte ich nun kurz eine Komposition ansprechen, die das Labyrinth im Titel trägt.

\section{Grosse Störungen in einem kleinen Labyrinth}

Die Zugehörigkeit des Kleinen harmonischen Labyrinths (BWV 591) zum CEuvre J.S. Bachs ist weder zu beweisen noch zu widerlegen. Seine drei Teile sind explizit bezeichnet: ein stark modulierender Introitus, ein als dreistimmige Fuge konzipiertes Centrum und ein ebenfalls stark modulierender Exitus. Die beiden äusseren Teile zeichnen einen Pfad im Medium des Quintenzirkels: Der Introitus moduliert über verschiedene Stationen von C-Dur über Fis-Dur, der im Quintenzirkel C-Dur entgegengesetzten Tonart, nach c-Moll; der Exitus von C-Dur über u.a. Fis-Dur nach C-Dur.

Man könnte aufgrund dieser symmetrischen und daher alles andere als verwirrenden Disposition erwarten, dass es sich nicht um ein echtes Labyrinth handelt, sondern nur um die Darstellung der Architektur eines Labyrinths. Dies stimmt aber nur, solange man aus der ex-post-Perspektive gleichsam von oben in dieses Labyrinth hineinblickt. Schreitet man ex ante hörend in diesen Klangraum hinein, wird man in ein echtes Labyrinth verstrickt. Der Introitus zeigt dies sehr schön: In den ersten sechs Takten ist man mit einem durch Dominante und Subdominante abgesicherten C-Dur harmonisch auf festem Boden. Doch ab T.7 werden die Konsequenzen hörbar, die aus der Anwendung der ersten beiden Methoden labyrinthischer Desorientierung resultieren:

(1.) Der Entzug von markanten Bezugspunkten geschieht T.7ff nicht nur durch den Verzicht auf Kadenzen, sondern auch durch die Häufung von dreizehn verminderten Septakkorden in zehn Takten. Bei diesen je aus vier kleinen Terzen bestehenden Akkorden gibt es keinen Grundton. Weil so jeder seiner Töne zum Leitton erklärt werden kann, ist es unmöglich, von ihm eindeutig auf eine harmonische Ordnung $\mathrm{zu}$ rekurrieren und damit ex ante $\mathrm{zu}$ bestimmen, wohin sich die Modulation fortentwickelt. Dies wird durch die rhythmi- 
sche Brechung der Akkorde und die häufigen Überbindungen und Vorhalte verschärft, weil die Akkorde so nicht separiert nacheinander erklingen, sondern in einem ständigen Prozess des Auflösens und neu Zusammenfügens auseinander hervorgehen.

(2.) Eine Setzung von irreführenden Bezugspunkten liegt mit den häufigen enharmonischen Verwechselungen vor. So wird z.B. auf der zweiten Zählzeit von T.7 der aus T.6 im Bass übergehaltene Grundton $\mathrm{C}$ enharmonisch zum His umgedeutet, womit er als Leitton innerhalb von cis-Moll fungiert. Das lesende Auge mag hier noch folgen können, doch das Ohr kommt bei dieser unhörbaren Umdeutung zwangsläufig zu spät, zumal cis-Moll im Folgenden harmonisch nicht stabilisiert wird.

Der Exitus operiert in vergleichbarer Weise, weshalb ich ihn hier übergehe und sogleich zum Centrum mit seiner dreistimmigen Fuge fortschreite. Diese ist recht konventionell und betreibt keine Eskalation des Orientierungsbedürfnisses. Auch ist sie zu harmlos, um sie als Darstellung des Kampfes mit dem Minotaurus deuten zu können. Und auch die Notenfolge B-A-C-H im vorletzten Takt hilft nicht weiter - ist es eine Hommage oder eine Signatur? Wie auch immer: Ich vermute, dass das Centrum einen bisher ungenannten Aspekt musikalischer Labyrinthik beleuchtet. Diesem sei ein eigener Abschnitt gewidmet.

\section{Fugen, Labyrinthe und Ariadnen}

Fugen haben eine unbestreitbare Affinität zum Thema 〈Orientierung〉 - dies zeigt sich schon daran, dass die Fugenlehre ariadnische Figuren wie dux und comes kennt. Komponist, Interpret und Hörer dürfen im komplexen Klangraum der Fuge die Orientierung nicht verlieren, und zwar einerseits im Hinblick auf die selbständigen Stimmen und ihren Verlauf, andererseits bezüglich des Themas und seiner mitunter von fugentechnischen Metamorphosen begleiteten Wanderung durch die Stimmen. Allzu leicht kommt es zu kompositorischen Regelverstössen, interpretatorischen Fehlgewichtungen und Überforderungen des Hörers.

Eine Fuge gilt als umso kunstvoller, je komplexer das Material disponiert und je raffinierter dessen Ausgestaltung bewerkstelligt ist - oder anders: je mehr sie die Orientierungskompetenz fordert, ohne sie zu überfordern. Die Rekurse von Bezugspunkten auf musikalische Ordnungen, sollen demnach gerade noch möglich sein. Damit steht die Fuge den musikalischen Labyrinthen überaus nahe. Beide 
spielen mit dem Orientierungsvermögen, aber während das eine auf die Verwirrung hinaus will, hat es die andere auf die gerade noch gelingende Orientierung abgesehen. Aus diesem Grund hausen in der Fuge als Gattung irritierend viele Ariadnen: geltende Gesetze, die an jeder Ecke gegenwärtig sind und befolgt werden wollen. Im Labyrinth hingegen ist die Ariadne eine erhoffte, mythisch-spärliche Möglichkeit.

Indem beide, das Labyrinth und die Fuge, auf den schmalen Grad zwischen gelingender und misslingender Orientierung verweisen, verdichten sie symbolisch das unhintergehbare Angewiesensein des Menschen auf gelingende Orientierung. Damit berühren sie eine religiöse Sphäre: Nicht zufällig ist in den Boden der Kathedrale von Chartres ein Labyrinth als Mosaik eingezeichnet, und nicht ganz zufällig ist die Fuge eine bevorzugte Gattung der Kirchenmusik. Beide sind gleichsam begehbare Symbole, in denen das Symbolisierte performativ erfahren wird. Beide betonen dabei einen anderen Aspekt: Das Labyrinth symbolisiert das Leben als einen spielerisch-offenen Orientierungsraum, in dem man sich einerseits frei bewegen kann, aber andererseits auch ganz auf sich allein gestellt ist. Die Kunst des Labyrinths ist die Kunst, selbständig Ordnungen zu finden, die im Leben orientieren und damit einen gelingenden Weg eröffnen. Wem dies nicht gelingt, der wird zur Beute des Minotaurus. Die Fuge wiederum symbolisiert das Leben als einen Orientierungsraum, der von unverrückbaren Ordnungen bestimmt ist. Orientierung im Leben gelingt nur mit Hilfe dieser Ordnungen. Die Kunst der Fuge ist die Kunst, die richtigen Ordnungen zu kennen und auf sie zu rekurrieren. Wem das nicht gelingt, der kann vor dem strengen Richter des Gesetzes nicht bestehen. Pointiert gesagt: Im Labyrinth ereignet sich gelingende Orientierung als eine ariadnische Gabe, die dem Orientierungssubjekt erhofft oder unverhofft zuteil wird; im autoritativ regierten Raum der Fuge gibt es sie hingegen nur als Gehorsam.

Damit kann das Nebeneinander von Labyrinth und Fuge im Kleinen harmonischen Labyrinth als Hinweis auf beide Aspekte gelingender Orientierung gedeutet werden. Daran anschliessend müsste man die Position der Fuge in BWV 591 nicht nur deskriptiv dahingehend interpretieren, dass die geltenden Normen faktisch im Centrum der Komposition bzw. des Komponierens insgesamt stehen, sondern auch normativ dahingehend, dass sie grundsätzlich im Zentrum des Lebens zu stehen hätten. Aber ist dieser Anspruch auch in nachaufklärerischen Zeiten noch haltbar? Es wird Zeit, den Klangraum der Grande Fugue zu betreten. 


\section{Der Schiffbruch der Orientierung in der Grande Fugue}

Beethovens Opus 133 ist weder ein musikalisches Labyrinth, noch eine Fuge im strengen Sinn, denn es gibt weder Hinweise, dass der Hörer intentional desorientiert werden soll, noch ist das Formprinzip der Fuge konsequent durchgehalten. Und doch kommt es in diesem Klangraum zu Eskalationen des Orientierungsbedürfnisses. Schon ein Rezensent der Uraufführung musste eingestehen: «Aber den Sinn des fugirten Finale wagt Ref. nicht zu deuten: für ihn war es unverständlich wie Chinesisch» - und im Folgenden wird zur Erklärung nicht nur das Fremde, sondern auch das Illegale, Pathologische oder sprichwörtlich Babylonische herbeizitiert. Wie kommt es zu diesem Schiffbruch?

(1.) In formaler Hinsicht ist die Grande Fugue durchsetzt von mehrdeutigen und damit irreführenden Bezugspunkten. Wie man bei Gerd Indorf genauer nachlesen kann, besitzt sie die statisch-strengen Eigenschaften der 〈Fuge〉 ebenso wie die prozessual-variablen der 〈Variation〉 oder die weiträumig-kontrastierenden der 〈Sonate〉 bzw. des 〈Sonatenzyklus〉. ${ }^{3}$ Dies schlägt sich nicht zuletzt im sperrigen Aufbau des Stückes nieder. Ich übernehme die Einteilung Indorfs:

1. Overtura (T.1-30): Vorstellung einer Art Fugenthema in vierfacher Gestalt (Einführung in der umgekehrten Reihenfolge des späteren Auftretens)

2. B-Dur-Fuge (T.30-158)

3. Ges-Dur-Fugato (T.159-232)

4. 〈Alla marcia>-Episode (T.233-272)

5. As-Dur-Fuge (T.273-314)

6. Reprisenelemente der B-Dur-Fuge (T. 414-492)

7. Reprisenelemente des Ges-Dur-Fugato (T.493-510)

8. Überleitung und Rückkehr nach B-Dur (T.511-532)

9. Reprise der 〈Alla marcia〉-Episode (T.533-564)

10. Satzcoda (T.565-714)

Je nachdem wie man diese Segmente gewichtet und kombiniert, ergibt sich eine Affinität zu der einen oder anderen Ordnung, man könnte sie etwa zu vier Sätzen einer Sonate zusammenfassen. Die für die verschiedenen Formen essentiellen Merkmale, werden dabei nicht nur additiv gereiht, sondern bisweilen auch integriert. So

G. Indorf, Beethovens Streichquartette, Freiburg i.Br. 2004, 431ff. 
präsentiert sich den Hörern ein schillerndes Gebilde, das sich einer einfachen Ortung entzieht.

(2.) Auch die Melodik ist geeignet, die Orientierung zu erschweren. Dies lässt sich von der Intervallfolge her verstehen, die in der Overtura in vierfacher Gestalt eingeführt wird und in der sich die Töne massiv am oberen und unteren Ende eines Oktavraums ballen: g', gis', a' und e", f", fis", g" (T.2ff,Violine I). Auffällig sind die in der Mitte klaffende Leerstelle (b bis dis) und die Vermeidung der zum G-Dur-Dreiklang gehörigen Töne h und d. Diese Disposition führt zusammen mit der Neigung zu Chromatik und Leittönigkeit - dazu, dass die harmonische Ortung melodischer Strukturen oft ungewiss bleibt. Zudem sind in ihr melodisch sowohl Sprünge zwischen den beiden Tonzentren (etwa im Kontrasubjekt T.30ff), als auch (wenn beide Zentren aneinandergerückt werden) ein kleinschrittiges Zirkulieren (etwa die Sechzehntelbewegungen T.159ff) angelegt. So bestehen keine guten Ausgangsbedingungen für die Genese melodischer Markanz.

(3.) In der Rhythmik könnten sich viele Strukturen aufgrund ihrer Pointierung und Stabilität als markante Bezugspunkte beschreiben lassen. Doch oft sind sie so hartnäckig stabil, dass ihre Markanz ins Redundante kippt. Zudem sind oft so viele Ebenen übereinander gelegt, dass man als Hörer schlicht nicht mehr weiss, worauf man seine Aufmerksamkeit lenken soll. Orientierungsvollzüge ersticken so an einem Überangebot von möglichen Bezugspunkten.Verstärkt wird dieser Effekt durch das allgegenwärtige Insistieren auf klangliche Intensität.

Form, Melodik und Rhythmik der Grossen Fuge sind demnach geeignet, den Hörer zu desorientieren. Dieses Eskalationspotential teilt sie mit dem Spätwerk Beethovens insgesamt, von dem Friedhelm Krummacher schreibt, es ziele auf eine «Synthesis des Disparaten $»^{4}$ und zu dem Adorno in seinen Beethovenfragmenten äussert, «Dissoziation und Zerfall» würden hier «zum Kunstmittel». ${ }^{5}$ Ist Beethoven in seinem Opus 133 ganz einfach zu weit gegangen? Hat er ohne zu wollen eine anarchische Fuge oder ein auswegloses Labyrinth geschaffen?

Ich möchte abschliessend eine Strategie skizzieren, mit der Orientierung in der Grande Fugue trotz allem gelingen kann. Der Schlüssel dazu liegt in der Overtura, die wesentlich mehr ist als ein

\footnotetext{
4 F. Krummacher, Synthesis des Disparaten. Zu Beethovens späten Quartetten und ihrer frühen Rezeption, in: Archiv für Musikwissenschaft 37/2 (1980), 99-134.

5 T.W.Adorno, Beethoven. Philosophie der Musik, Nachgelassene Schriften I,1, hg.v. R. Tiedemann, Frankfurt a.M. ${ }^{3} 1999,267$.
} 
lapidar präsentierter Katalog des thematischen Materials wie es Indorf nahe legt.

\section{Tantôt libre - tantôt recherchée}

«Eine Fuge zu machen [...] ist keine Kunst, ich habe deren zu Dutzenden in meiner Studienzeit gemacht. Aber die Phantasie will auch ihr Recht behaupten, und heut' zu Tage muß in die alt hergebrachte Form ein anderes, ein wirkliches poetisches Element kommen.» Ludwig van Beethoven

Zunächst muss man die grundsätzliche Spannung verstehen, unter der die Grande Fugue steht. Beethoven bezeichnet die Pole im Untertitel mit tantôt libre und tantôt recherchée. Dies ist keine vorweggenommene Rechtfertigung gegenüber pharisäischen Einwänden, sondern eine orientierungsstrategische Anweisung an Interpreten und Hörer. Diese werden darauf hingewiesen, dass sie nun einen Raum betreten, in dem die Freiheit ebenso möglich ist wie der Gehorsam. Mit dieser Deutung knüpfe ich an die beiden oben genannten Aspekte menschlichen Sich-Orientierens in der Welt an, wie sie durch Labyrinth und Fuge symbolisiert werden konnten: Im Labyrinth gelingt Orientierung, indem das Subjekt angesichts eines Freiheitsraumes kreativ nach Ordnungen sucht, im autoritativ regierten Raum der Fuge, indem es geltende Ordnungen anerkennt. Aber was in BWV 591 fein säuberlich getrennt war, ist in Opus 133 dialektisch synthetisiert. Orientierung gelingt hier nur dem, der mit der eingeräumten Freiheit ebenso umzugehen weiss, wie mit der Notwendigkeit, auf vorgegebene Ordnungen zu rekurrieren. Ein Klangraum, der dies zulässt, muss in besonderer Weise beschaffen sein. Beethoven geht dazu von der formalen Grundidee der `Fuge aus und verändert diese radikal.

Üblicherweise wird in den ersten Takten einer Fuge ein charakteristisches und stabiles Thema exponiert. Eine solche Exposition erfüllt die Funktion, eine Ordnung zu definieren, auf die im Laufe des Stücks angesichts geeigneter Bezugspunkte rekurriert werden kann. Tatsächlich präsentiert die Overtura eine charakteristische Intervallfolge; doch tut sie dies in einer hochkomplexen Gestalt: 

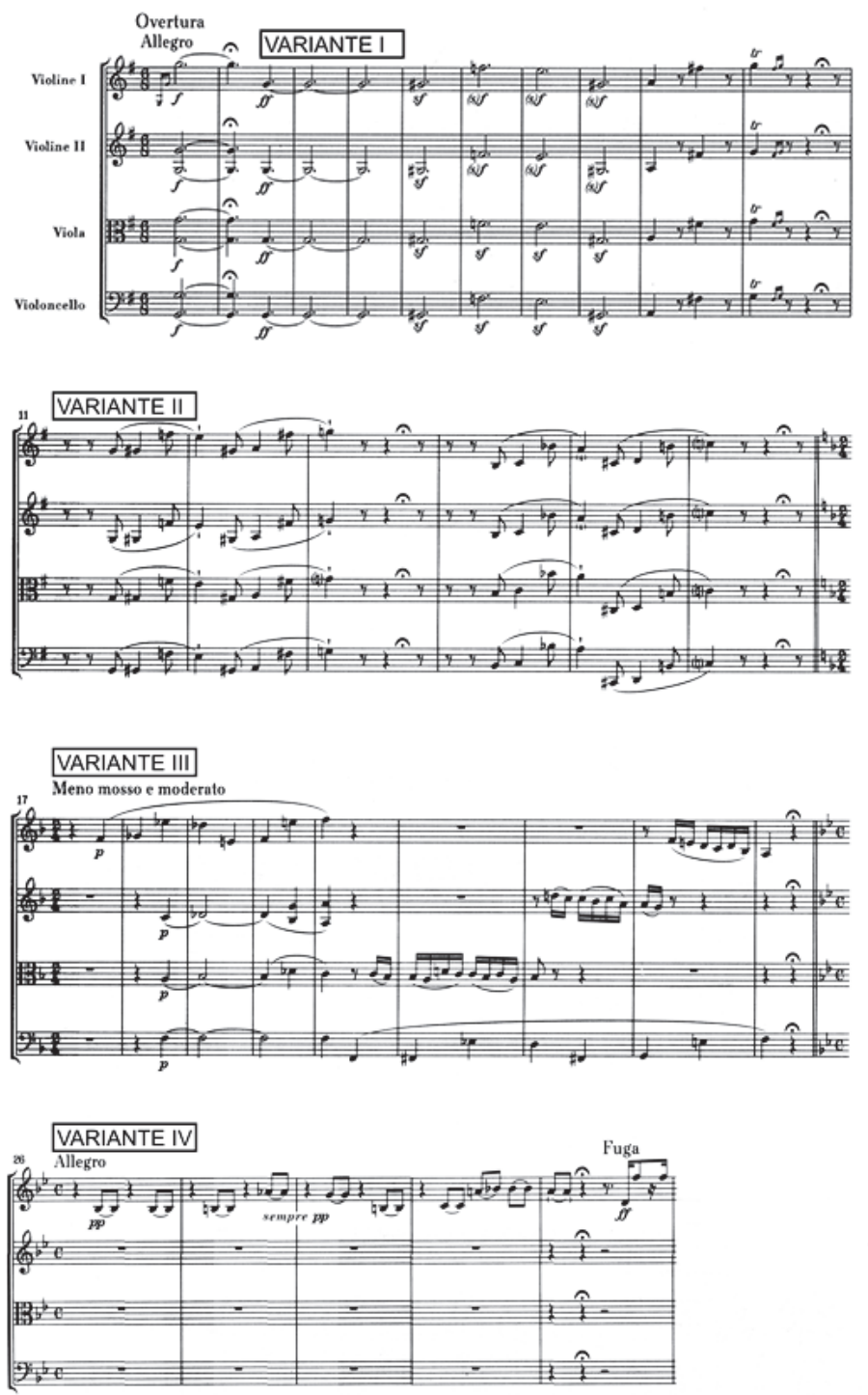

Beethoven op. 133, T.1-30 (Copyright 2007 G. Henle Verlag. Abdruck mit freundlicher Genehmigung) 
Anstelle der klaren und distinkten Definition eines Fugenthemas präsentiert Beethoven ein Geflecht aus verschiedensten Elementen, Beziehungen und Prozessen. Nur wenige Beobachtungen: Aufs Ganze gesehen ergibt sich ein gross angelegter Spannungsbogen von Variante I zu IV, wobei der Höhepunkt am Ende von II liegt.Variante II geht dabei organisch aus I als Beschleunigung und rhythmische Eskalation hervor, wobei sich in II zudem eine Verdoppelung ereignet, die sich aufgrund der Generalpause ebenso als vertikale Spaltung verstehen lässt.Variante III kontrastiert gegenüber I und II durch die Anweisung Piano, eine neue Tonart sowie ein deutlich verlangsamtes Metrum; sie übernimmt gleichwohl die Spaltung aus II und überträgt sie auf die horizontale Ebene (ein führendes Instrument vs. drei Begleitstimmen), woraus eine chiastische Struktur innerhalb von III resultiert. Beide Gestalten der Begleitung operieren imitatorisch mit isolierten Elementen der charakteristischen Intervallfolge, wobei die erste an die langsamere Variante I, die zweite an die beschleunigte Variante II anknüpft. Mit Variante IV wechseln erneut Tonart und Metrum; es kommt zu einer Rückkehr zur Bezeichnung Allegro aus I und II. Gegenüber III ist die Lautstärke ins Pianissimo weiter abgesenkt. Die in II begonnene Isolation einzelner Elemente wird weitergefuhrt und geht in einen Zersetzungsprozess über: Die erste Violine spielt die Intervallfolge ganz allein und zudem in einer brüchigen Gestalt, was durch eine Separierung der einzelnen Töne mittels Pausen erzielt wird. Die Notierung der Viertel in je zwei übergebundenen Achteln markiert eine innere Instabilität. Anders als zu erwarten, endet Variante IV nicht auf b, sondern auf a. Es wird also ein zusätzlicher fallender Sekundschritt additiv hinzugefügt, was eine Infragestellung bzw. Destabilisierung erstens derVariante IV, zweitens der gesamten Overtura und damit drittens auch der ihr zu Grunde liegenden Intervallfolge impliziert.

Schon diese knappen Bemerkungen genügen, um die Vielschichtigkeit dieses Gefüges zu erahnen. Beethoven übernimmt von der Gattung 〈Fuge〉 die Idee der vorgängigen Definition einer musikalischen Ordnung, gestaltet diese aber komplex und offen bzw. instabil. Dies gibt Beethoven die Möglichkeit, im Verlauf des Stückes einige Elemente im Sinn strenger Fugengesetze zu gestalten, auf andere aber frei zurückzukommen und spielerisch weiterzuentwickeln. Beethoven entwirft damit eine neue inkludierende Gattung, die nur noch in Anführungsstrichen als ‘Fuge〉 bezeichnet werden kann.

Während dem Hörer normalerweise schon durch die Nennung der Gattung eine bestimmte Orientierungsstrategie an die Hand gegeben wird, macht diese 〈Fuge` deutlich weniger Vorgaben. Ihr 
Klangraum lässt das spielerische Umherschweifen in den offenen Strukturen ebenso zu, wie das achtsame Verfolgen der streng gestalteten Prozesse. Der Hörer wird sich orientieren können, wenn es ihm gelingt, zwischen der Offenheit des Spiels und der Geschlossenheit der Fügsamkeit zu oszillieren. Dazu muss er sich die Overtura als erinnerte Ordnung präsent halten, um rekurrierend auf sie zurückkommen zu können - doch darf er sie nicht verabsolutieren, weil dies den Blick für die offenen Strukturen versperrt und notwendig Irritationen auslöst.

Wenn Labyrinth und Fuge je einen Aspekt menschlichen SichOrientierens in der Welt symbolisch verdichten konnten, so ist Beethovens ‘Fuge` in der Lage, beide Aspekte präsent zu halten. Ihr Klangraum kann daher als hörbares Symbol für das unhintergehbare Angewiesensein des Menschen auf gelingende Orientierung gedeutet werden - und zwar eines, das heutigen Situationen menschlichen Lebens angemessen ist. Denn um das Leben meistern zu können, müssen wir mit beidem rechnen: dass uns Freiheit zugebilligt bzw. abverlangt wird und dass wir mit faktisch geltenden Ordnungen konfrontiert werden. So wie das Leben verlangt auch die Grande Fugue mündige Subjekte, die in der Lage sind, sich flexibel ihrer eigenen Orientierungsfähigkeit zu bedienen. Sie ist darin ein zutiefst aufgeklärtes, ja modernes Stück. Unmündige Hörer werden hier zur leichten Beute des Minotaurus, doch auch mündige sind davor nie ganz gefeit.

Auch die christliche Theologie steht vor der schwierigen Aufgabe, zwischen dem Geltungsanspruch (religiöser) Ordnungen und der Möglichkeit freier Lebensgestaltung zu vermitteln. Wo diese Balance fehlt, drohen entweder die fundamentalistische Verabsolutierung religiöser Ordnungen oder der gänzliche Verzicht auf deren klare Definition und Explikation. Im ersten Fall kann Orientierung nur noch in reduziert komplexen Räumen gelingen, im zweiten ist sie gefährdet, weil diffuse Ordnungen zu unspezifisch sind, um reale Orientierungsbedürfnisse zu befriedigen. Es gilt, beides zu vermeiden, vielleicht im Anschluss an Beethovens Grande Fugue.

— Stefan Berg ist wissenschaftlicher Mitarbeiter am Collegium Helveticum Zürich und promoviert über das Verhältnis von Religion und Musik. 